\title{
Prevalence of abnormal SPECT myocardial perfusion imaging during the COVID-19 pandemic
}

\author{
Usman A. Hasnie ${ }^{1}$ - Pradeep Bhambhvani ${ }^{2}$ - Ami E. Iskandrian ${ }^{3}$ • Fadi G. Hage Pr, $^{3,4}$ \\ Received: 20 October 2020 / Accepted: 15 November 2020 / Published online: 8 January 2021 \\ (C) Springer-Verlag GmbH Germany, part of Springer Nature 2021
}

\begin{abstract}
Purpose The aim of this study is to evaluate the rate of abnormal myocardial perfusion imaging (MPI) studies at a single medical center during the COVID-19 pandemic compared to prior to the pandemic.

Methods We retrospectively studied stress single-photon emission computed tomography (SPECT)-MPI studies performed during the peak of COVID-19 restrictions at the University of Alabama Medical Center in comparison to the same time period in 2019. Results SPECT-MPI volume was reduced from 553 per month in 2019 to 105 per month in 2020. The proportion of abnormal SPECT-MPI for the 2020 cohort ( $61 \pm 13$ years, $48 \%$ men, $41 \%$ black) was not different from the 2019 cohort (62 \pm 12 years, $48 \%$ men, $42 \%$ black) ( $31 \%$ vs. $27 \%, p=0.4)$. Similar proportion of patients in the 2 cohorts had abnormal myocardial perfusion, moderate-large perfusion defects, myocardial ischemia, myocardial scar, and abnormal left ventricular ejection fraction. The proportion of abnormal SPECT-MPIs was not different based on whether patients were evaluated face-to-face or by telemedicine ( $28 \%$ vs. $27 \%, p>0.9$ ) but was higher for cardiology providers ( $40 \%$ vs. $20 \%, p<0.001)$.

Conclusions There was a significant reduction in the number of SPECT-MPI studies performed during the peak restrictions from the pandemic. Despite this restriction, the rate of abnormal studies remained stable. Our study suggests that it remains difficult to predict which patients will have abnormal SPECT-MPI even when providers and stress laboratories are forced to prioritize the performance of studies to high-yield patients.
\end{abstract}

Keywords Myocardial perfusion imaging $\cdot$ COVID-19 $\cdot$ Telehealth

Subject terms list Imaging $\cdot$ Nuclear cardiology and PET $\cdot$ Ischemia

Key Points In this retrospective cohort study, there was an expected reduction in the volume of SPECT-MPI studies completed during the COVID-19 pandemic. However, the prevalence in abnormal SPECTMPI studies in $2020(31 \%)$ versus 2019 (27\%) was not significantly different. It remains challenging to predict which patients will have an abnormal SPECT-MPI study even when facilities attempt to prioritize completion of imaging studies to high-yield patients.

This article is part of the Topical Collection on Cardiology

Fadi G. Hage

fadihage@uab.edu

1 Department of Medicine, University of Alabama at Birmingham, Birmingham, AL, USA

2 Division of Molecular Imaging and Therapeutics, Department of Radiology, University of Alabama at Birmingham, Birmingham, AL, USA
3 Division of Cardiovascular Disease, Department of Medicine, University of Alabama at Birmingham, LHRB 326, 701 19th Street South, Birmingham, AL 35294, USA

4 Section of Cardiology, Birmingham Veterans Affairs Medical Center, Birmingham, AL, USA 


\section{Introduction}

The novel severe acute respiratory syndrome coronavirus 2 (SARS-CoV-2) which initially emerged in Wuhan, China, in December 2019, quickly progressed to a global pandemic [1]. As of November 2020, more than 50 million patients had contracted the disease worldwide, including more than 10 million in the USA, according to the Johns Hopkins COVID-19 dashboard [2]. The Centers for Disease Control and Prevention (CDC) reported over 200,000 deaths in the USA [3]. As such, the COVID-19 pandemic impacted medical care in the USA and throughout the world. In addition to dealing with surges in cases amid shortages of personal protective equipment, healthcare systems had to ration care in order to mitigate the spread of the pandemic and protect patients seeking care for other indications from contracting the virus. This resulted in marked reductions in "non-essential" medical care including cardiovascular procedures and imaging $[4,5]$. In one study, there was a reduction in ST segment elevation myocardial infarction (STEMI) cases as measured by a $38 \%$ reduction in STEMI activations across 9 high volume cardiac catheterization laboratories [6]. This reduction in STEMI cases and hospitalizations was supported by other studies in the USA and globally [7-9].

The American Society of Nuclear Cardiology (ASNC) in collaboration with the Society of Nuclear Medicine and Molecular Imaging (SNMMI) issued guidance regarding postponement of all non-urgent nuclear cardiology studies during the pandemic $[10,11]$. This resulted in significant restrictions on the performance of stress single-photon emission computed tomography (SPECT) myocardial perfusion imaging studies (MPI) nationally, although the extent of this restriction has not been reported yet. Over the last few decades, there has been a decline in the rate of abnormalities detected on SPECT-MPI $[12,13]$. It has been proposed that one source for this decline is the referral of lower risk patients for testing [14]. We therefore hypothesized that during the peak of the restrictions from the pandemic, when our medical center was able to perform far fewer SPECT-MPIs than usual, the rate of abnormal SPECT-MPI will increase due to better selection of patients referred for testing. Since some studies have suggested a relationship between referral patterns and appropriateness of SPECT-MPI, we also evaluated whether such a shift in the rate of abnormal SPECT-MPI may be related to change in referral patterns $[15,16]$.

During the pandemic, there was a national and international surge in the use of telemedicine to evaluate patients remotely without face-to-face contact using technologies (audio and/or video conferencing) that have not been utilized on such a wide scale previously $[17,18]$. While the use of telemedicine facilitated the provision of care during the pandemic and may well find expanding usage after the pandemic as a way to extend the geographic reach of cardiologists in specific scenarios, it is not currently known whether evaluating patients using telemedicine is as effective as in-person evaluation for identifying patients who are more likely to have abnormal SPECT-MPI. We therefore also hypothesized that the rate of abnormal SPECT-MPI will be lower in patients evaluated using telemedicine.

\section{Methods}

At the University of Alabama at Birmingham Medical Center, the peak of restrictions from the COVID-19 pandemic on MPI occurred in April and May 2020 with gradual easement of these restrictions afterwards. We therefore retrospectively identified all patients who underwent stress single-photon emission computed tomography (SPECT)-MPI for all indications at our medical center from April 1 to May 31, 2020. As a control group, we identified the corresponding cohort during the same time period in 2019, well prior to the pandemic, and included every third patient. In the event that data was missing for a patient, an adjacent patient on the list was included instead. Baseline demographics, past medical history including comorbidities, medication intake, referring provider specialty, and visit type (whether by telemedicine or face-to-face), and indication for and findings on SPECT-MPI were retrieved from the electronic medical record.

Patients underwent SPECT-MPI using standard protocols according to ASNC guidelines [19]. We have previously published details regarding the performance and interpretation of SPECT-MPI at our laboratory [20-24]. Perfusion abnormalities were identified and characterized using a quantitative software program (Corridor4DM) with visual supervision [25]. Left ventricular (LV) ejection fraction (EF) was determined from gated images [26]. Abnormal LVEF was defined as LVEF $<50 \%$. Perfusion defects were quantitated as percent of LV myocardium and expressed as total perfusion defect size (PDS), ischemia (reversible perfusion defect), and scar (fixed perfusion defect). Abnormal perfusion was defined as PDS $\geq 5 \%$ LV mass. An abnormal SPECT-MPI was defined as the presence of abnormal perfusion and/or abnormal LVEF.

\section{Statistical analysis}

Continuous variables were presented as mean \pm standard deviation (normal distribution) or median and interquartile range (IQR) and compared between the groups using the unpaired $t$ test or Mann-Whitney $U$ test, as appropriate. Categorical variables were expressed as frequencies (percentage) and compared using the Pearson chi-square test or Fisher's exact test. All tests were two-tailed, and a $p$ value $<0.05$ (set a priori) was considered statistically significant. All statistical analyses and graphics creations were performed using SPSS Statistics version 25 software (IBM Corp., Armonk, NY, USA). 
Table 1 Baseline characteristics

\begin{tabular}{llll}
\hline & $2019(n=368)$ & $2020(n=210)$ & $p$ value \\
\hline Age (years) & $62 \pm 12$ & $61 \pm 13$ & 0.2 \\
Male gender & $178(48 \%)$ & $101(48 \%)$ & $>0.9$ \\
Race & & & 0.6 \\
Caucasian & $206(56 \%)$ & $119(57 \%)$ & \\
Black & $156(42 \%)$ & $85(41 \%)$ & \\
Other & $6(2 \%)$ & $6(3 \%)$ & 0.09 \\
Diabetes & $139(38 \%)$ & $64(31 \%)$ & 0.2 \\
Hypertension & $274(75 \%)$ & $166(79 \%)$ & 0.08 \\
Dyslipidemia & $194(53 \%)$ & $127(61 \%)$ & $<0.001$ \\
ESRD & $57(16 \%)$ & $7(3 \%)$ & 0.006 \\
Heart failure & $36(10 \%)$ & $38(18 \%)$ & 0.5 \\
Myocardial infarction & $39(11 \%)$ & $18(9 \%)$ & 0.1 \\
Coronary revascularization & $59(16 \%)$ & $45(21 \%)$ & 0.1 \\
CABG & $22(6 \%)$ & $21(10 \%)$ & 0.2 \\
PCI & $45(12 \%)$ & $35(17 \%)$ & 0.001 \\
Current tobacco use & $47(13 \%)$ & $50(24 \%)$ & 0.001 \\
Referring provider & $40 \%$ & $47 \%$ & \\
Cardiology & $37 \%$ & $43 \%$ & $6 \%$ \\
Primary care/internal medicine* & $19 \%$ & $4 \%$ & \\
Transplant & $4 \%$ & & \\
Surgery & & & \\
\hline
\end{tabular}

Baseline characteristics of patients who underwent MPI during the peak of the restrictions in 2020 and a control group from a similar time period in 2019

*Including internal medicine subspecialties

$C A B G$ coronary artery bypass graft, $P C I$ percutaneous coronary intervention

Table 2 Medication list

\begin{tabular}{lccl}
\hline & $2019(n=368)$ & $2020(n=210)$ & $p$ value \\
\hline Aspirin & $157(43 \%)$ & $85(41 \%)$ & 0.6 \\
Clopidogrel & $30(8 \%)$ & $20(10 \%)$ & 0.6 \\
Beta-blocker & $159(43 \%)$ & $96(46 \%)$ & 0.6 \\
ACE-I/ARB & $145(40 \%)$ & $80(38 \%)$ & 0.8 \\
CCB & $125(34 \%)$ & $69(33 \%)$ & 0.8 \\
Loop diuretic & $117(32 \%)$ & $44(21 \%)$ & 0.005 \\
Nitrates & $33(9 \%)$ & $30(14 \%)$ & 0.05 \\
Statin & $161(44 \%)$ & $113(53 \%)$ & 0.02 \\
Insulin & $41(11 \%)$ & $21(10 \%)$ & 0.8 \\
Oral hypoglycemic & $59(16 \%)$ & $46(22 \%)$ & 0.09 \\
\hline
\end{tabular}

Relevant medication among patients who underwent MPI during the peak of the restrictions in 2020 and a control group from a similar time period in 2019

$A C E-I$ angiotensin-converting enzyme inhibitor, $A R B$ angiotensin II receptor blocker, $C C B$ calcium channel blocker

\section{Results}

The 2020 cohort consisted of 210 patients who underwent SPECT-MPI between April 1 and May 31, 2020. During a similar time period in 2019, 1106 patients underwent SPECTMPI at our institution. Therefore, the SPECT-MPI volume at our institution dropped from an average of 553/month to 105/ month, i.e., to $19 \%$ of regular utilization during the peak of the restriction.

The baseline characteristics of the two cohorts are shown in Table 1 . The average age of patients in the 2020 cohort was 61 years, $48 \%$ were men, and $41 \%$ were black. There was a significant prevalence of coronary artery disease (CAD) risk factors and comorbidities, and 1 in 5 had prior coronary revascularization. Compared to the 2019 cohort, there was a higher prevalence of heart failure and current tobacco use and a lower prevalence of end-stage renal disease (Table 1). The use of relevant medications at time of SPECT-MPI is shown in Table 2. In the 2020 cohort, more patients were taking statins and less were taking loop diuretics compared to the 2019 cohort. The use of nitrates was borderline higher in the 2020 cohort. 
During April and May of 2020, there was a significant decrease in the proportion of tests done in the outpatient setting compared to 2019 (64\% vs. $79 \%, p<0.001)$. There was also an increase in the proportion of tests done with pharmacologic stress (regadenoson, $80 \%$ vs. $72 \%, p=0.04$ ). A significant proportion of SPECT-MPIs in the 2020 cohort were ordered based on telemedicine evaluation $(31 \%)$, while telemedicine was not used for this purpose in 2019. More studies in the 2020 cohort were referred from cardiology or primary care/internal medicine providers and less from transplant specialists than in 2019 (Table 1). With regard to the indication for performing SPECT-MPI, there was a decrease in the proportion of studies done for pre-operative evaluation (19\% vs. $29 \%, p=0.009$ ) and an increase in those done for evaluation of chest pain $(58 \%$ vs. $32 \%, p<0.001)$, shortness of breath $(31 \%$ vs. $16 \%, p<0.001)$, and heart failure $(7 \%$ vs. $3 \%, p=$ 0.03 ) in the 2020 cohort. Studies done after recent acute coronary syndrome ( $9 \%$ vs. $2 \%, p=0.001$ ) and for evaluation of typical angina ( $13 \%$ vs. $5 \%, p=0.002)$ were also more prevalent in 2020.

Findings on the resting ECG are shown in Table 3. In general, the 2 cohorts were comparable except for a trend towards more myocardial infarctions in the 2020 cohort that did not reach statistical significance. For patients who underwent exercise stress, the duration of exercise $(10 \mathrm{~min}$ (interquartile range 7-10 $\mathrm{min}$ ) in 2020 and $8 \mathrm{~min}(6-9 \mathrm{~min})$ in 2019, $p=0.2)$ and the effort achieved (10 METS (7-12 METs) in 2020 and 10 METs (7-10 METs) in 2019, $p=$ $0.9)$ were not different. The hemodynamic variables for both cohorts are shown in Table 4. For patients who exercised, there was no difference in the peak heart rate, and for those that underwent vasodilator stress, there was no difference in the heart rate response. During the stress test, the proportion of patients who experienced chest pain was similar (6\% vs. $3 \%$, $p=0.1)$. The ECG response to stress was also similar $(76 \%$ negative for ischemia, $19 \%$ non-diagnostic, and $5 \%$ positive for ischemia in 2020 compared to $78 \%, 16 \%$, and $6 \%$, respectively in $2019, p=0.6$ ).

Table 3 ECG findings

\begin{tabular}{llll}
\hline & $2019(n=368)$ & $2020(n=210)$ & $p$ value \\
\hline Sinus & $342(93 \%)$ & $201(96 \%)$ & 0.2 \\
Atrial fibrillation/flutter & $17(5 \%)$ & $4(2 \%)$ & 0.1 \\
Left bundle branch block & $9(3 \%)$ & $2(1 \%)$ & 0.3 \\
Paced rhythm & $8(2 \%)$ & $3(1 \%)$ & 0.8 \\
Myocardial infarction & $19(5 \%)$ & $19(9 \%)$ & 0.08 \\
Left ventricular hypertrophy & $16(4 \%)$ & $16(8 \%)$ & 0.1 \\
\hline
\end{tabular}

Findings on the resting ECG among patients who underwent MPI during the peak of the restrictions in 2020 and a control group from a similar time period in 2019
Of the SPECT-MPIs performed in the 2020 cohort, almost one-third were abnormal. This proportion was not different from that seen in 2019 ( $31 \%$ vs. $27 \%, p=0.4$ ). Similar proportion of patients had abnormal myocardial perfusion $(25 \%$ vs. $22 \%, p=0.4$ ), perfusion defect size $\geq 10 \%$ of LV myocardium $(16 \%$ vs. $19 \%, p=0.3)$, myocardial ischemia $(17 \%$ vs. $15 \%, p=0.6)$, myocardial scar ( $18 \%$ vs. $12 \%, p=0.08)$, and an abnormal LVEF (12\% vs. $13 \%, p=0.8)$ (Fig. 1). Among patients with abnormal myocardial perfusion, perfusion defect size (12\% of LV (8-22\%) in 2020 vs. $12 \%$ of LV (10-18\%) in $2019, p=0.4)$, extent of ischemia ( $5 \%$ of LV $(0-8 \%)$ in 2020 vs. $7 \%(0-11 \%)$ in $2019, p=0.07)$, and extent of scar $(8 \%$ of LV $(0-16 \%)$ in 2020 vs. $5 \%$ of LV $(0-10 \%)$ in $2019, p=0.1)$ were not different. Similarly, LVEF was not different between the cohorts $(65 \%(56-72 \%)$ in 2020 vs. $63 \%(55-72 \%)$ in 2019, $p=0.6)$.

The proportion of SPECT-MPIs that were abnormal was not different based on whether patients were evaluated faceto-face or by telemedicine ( $28 \%$ vs. $27 \%, p>0.9)$. These rates were also not different within the 2020 cohort based on evaluation method or for those evaluated face-to-face in 2020 vs. 2019 (Fig. 2). The proportion of abnormal SPECT-MPI was higher for patients referred from cardiology providers compared to other provider services $(40 \%$ vs. $20 \%, p<0.001$; Fig. 3). A similar trend was seen in the $2019(p<0.001)$ and $2020(p=0.003)$ cohorts, analyzed separately. However, the rate of abnormal SPECT-MPI was not different for each of the specialties between the 2020 and 2019 cohorts (Fig. 3).

\section{Discussion}

During the peak restrictions from the pandemic, the utilization of SPECT-MPI at our medical center dropped to less than $20 \%$ of baseline. This decrease was precipitated by multiple factors including (1) protecting patients from acquiring the infection in the medical setting, (2) protecting healthcare workers from acquiring the infection from patients presenting for testing, (3) preserving personal protective equipment, (4) diverting medical resources and personnel to deal with the pandemic surge, and (5) patient apprehension. Our center, in agreement with guidance from ASNC, delayed the performance of non-urgent testing and proceeded with performing tests only on patients who were considered to derive the most benefit from the test $[10,27]$. Consistent with this, there was a significant reduction in the percentage of studies that were done as an outpatient while in-patient evaluation was largely deemed as urgent.

We also noted a significant shift in the indication for SPECT-MPI during the pandemic from baseline. Significantly more studies were performed for symptom evaluation, and less studies were done for pre-operative evaluation which corresponded to a change in the referral pattern favoring studies 
Table 4 Hemodynamic variables

\begin{tabular}{llll}
\hline & 2019 & 2020 & $p$ value \\
\hline Exercise stress & $(n=263)$ & $(n=167)$ & \\
Baseline heart rate (beats/min) & $67(60-76)$ & $68(60-77)$ & $>0.9$ \\
Baseline systolic blood pressure (mm Hg) & $131(121-147)$ & $131(124-144)$ & 0.9 \\
Baseline diastolic blood pressure (mm Hg) & $78(72-87)$ & $75(69-82)$ & 0.1 \\
Peak heart rate (beats/min) & $149(138-163)$ & $152(143-164)$ & 0.4 \\
\% age-adjusted max & $93(88-100)$ & $90(87-101)$ & 0.4 \\
Peak systolic blood pressure (mm Hg) & $200(180-211)$ & $183(165-211)$ & 0.046 \\
Peak diastolic blood pressure (mm Hg) & $86(74-96)$ & $78(74-98)$ & 0.5 \\
Regadenoson stress & $(n=368)$ & $(n=210)$ & \\
Baseline heart rate & $70(63-79)$ & $73(65-81)$ & 0.1 \\
Baseline systolic blood pressure (mm Hg) & $135(118-149)$ & $133(119-146)$ & 0.3 \\
Baseline diastolic blood pressure (mm Hg) & $76(66-84)$ & $75(66-85)$ & $>0.9$ \\
Peak heart rate (beats/min) & $95(87-105)$ & $98(87-106)$ & 0.2 \\
Peak systolic blood pressure (mm Hg) & $140(123-159)$ & $140(124-159)$ & 0.8 \\
Peak diastolic blood pressure (mm Hg) & $74(63-83)$ & $70(62-82)$ & 0.1 \\
Heart rate response* & $32(22-46)$ & $32(20-45)$ & 0.7 \\
\hline
\end{tabular}

*Heart rate response is the percentage increase from baseline [16]

Hemodynamic variables during the stress test among patients who underwent MPI during the peak of the restrictions in 2020 and a control group from a similar time period in 2019

referred by cardiology and primary care or internal medicine vs. transplant services. This trend parallels a significant reduction in the volume of surgical cases reported from other medical centers [4]. SPECT-MPI is commonly used at our center for pre-transplant evaluation, which explains the lower prevalence of end-stage renal disease in the 2020 cohort (Table 1) [22, 28]. In accordance with guidance from ASNC, we noted a decrease in use of exercise stress in favor of pharmacologic stress to minimize droplet exposure to healthcare workers and the close contact between staff and patients, although this decrease was rather modest and we continued to perform exercise stress with appropriate precautions [10].
We anticipated an increase in the rate of abnormal SPECTMPI during the pandemic. We hypothesized that due to the highly restricted availability of testing, only high-yield patients will undergo SPECT-MPI, while those expected to be normal would be delayed. However, we did not find any statistically or clinically significant increase in the rate of abnormal SPECT-MPI or that of myocardial ischemia during this period (Fig. 1). We also did not see a significant increase in ischemic ECG changes during testing or a decrease in the heart rate response to vasodilator stress, both of which have been shown to correlate with prognostic outcomes [20, 29, 30]. A recent study by Megna et al. showed that pretest risk
Fig. 1 Bar graph comparing the proportion of abnormal SPECTMPI among patients who underwent MPI during the peak of the restrictions in 2020 and a control group from a similar time period in 2019

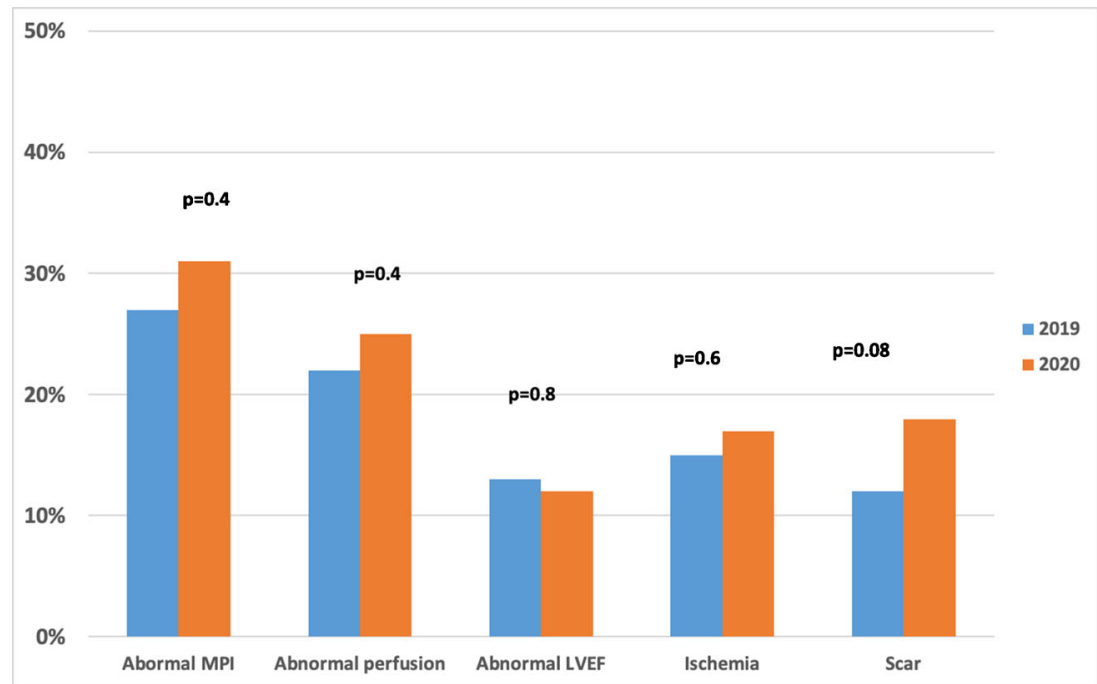


Fig. 2 Bar graph comparing the proportion of abnormal SPECTMPI among patients who underwent MPI during the peak of the restrictions in 2020 and a control group from a similar time period in 2019 based on whether their evaluation was face-to-face (F2F) or using telemedicine (tele). $p$ values are indicated on the figure

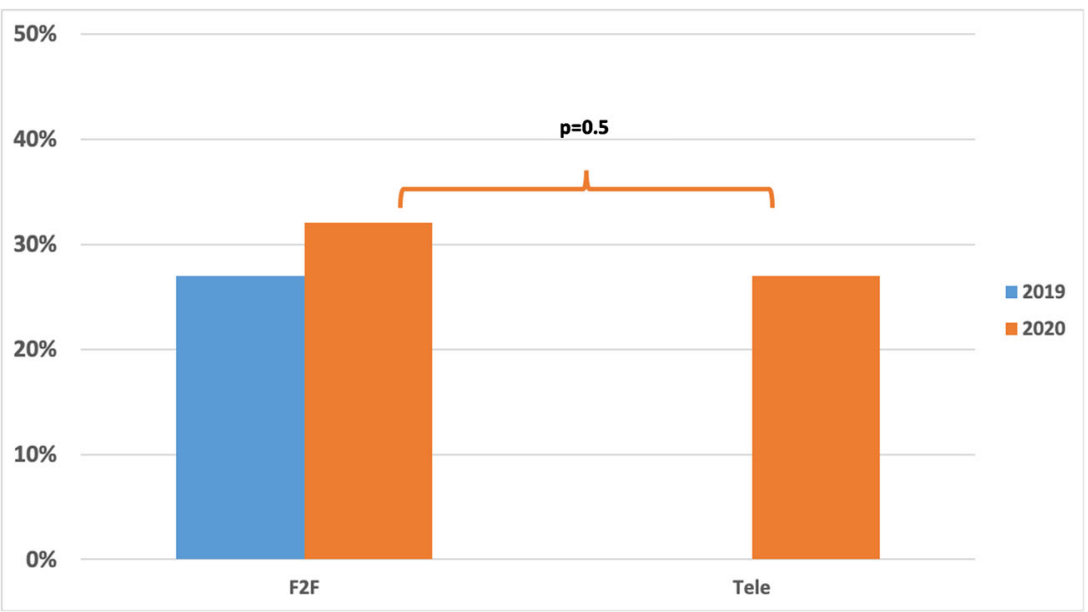

stratification based on cardiovascular risk factors has a limited value for predicting which patients will have an abnormal SPECT-MPI [31, 32]. Similarly, other studies that have attempted to use risk scores to predict abnormal SPECTMPIs have also demonstrated the limited abilities of clinical models to accurately predict the presence of myocardial ischemia without the use of imaging [33]. The current pandemic offered the opportunity to evaluate whether unmeasured variables that were not included in these models may influence their predictive abilities. The findings of our study suggest that even when incorporating unmeasured variables that were presumably considered by referring providers and nuclear cardiology laboratories, it remains difficult to predict which patients will have abnormal studies since the proportion of abnormal SPECT-MPI remained unchanged despite the significant restriction of access to the test.

As anticipated, a significant proportion of patients (almost one-third) were referred for SPECT-MPI based on telemedicine during the pandemic. However, we did not detect a significant effect of the mode of evaluation on the proportion of abnormal SPECT-MPI, and therefore, the use of telemedicine is not likely to be the reason that we did not detect an increase in abnormal SPECT-MPIs during the pandemic although further studies are needed in this area. Finally, we detected a shift in the referral pattern during the pandemic towards more studies being ordered by cardiology providers. However, since this shift occurred in the same direction favoring abnormal SPECT-MPIs (studies ordered by cardiology providers were 2-fold as likely to be abnormal compared to non-cardiology providers), the change in the referral pattern is an unlikely cause for not detecting an increase in abnormal SPECT-MPIs.

Recently, Nappi et al. investigated the rates of abnormal stress SPECT-MPI studies in a single-center study in Italy. Similar to our experience, they noted a significantly lower number of studies during the pandemic compared to the mean number in the corresponding months in 2017 to 2019 (123 vs. 413 , or $30 \%$ of regular utilization compared to $19 \%$ for our center). Similar to our experience, they reported no difference in the percentage of abnormal studies between the 2 time periods ( $36 \%$ vs. $34 \%, p=0.65$ ) [34]. This study further validates our results demonstrating no difference in the rate of abnormal SPECT-MPI study results during the COVID-19 pandemic compared to the pre-pandemic cohort. Further, Nappi et al. point out that given the lower number of studies
Fig. 3 Bar graph comparing the proportion of abnormal SPECTMPI among patients who underwent MPI during the peak of the restrictions in 2020 and a control group from a similar time period in 2019 based on the referring provider (cardiology, primary care $(\mathrm{PC})$ /internal medicine (IM), transplant, or surgery). Comparisons between the 2019 and 2020 cohorts for each specialty as denoted by each $p$ value

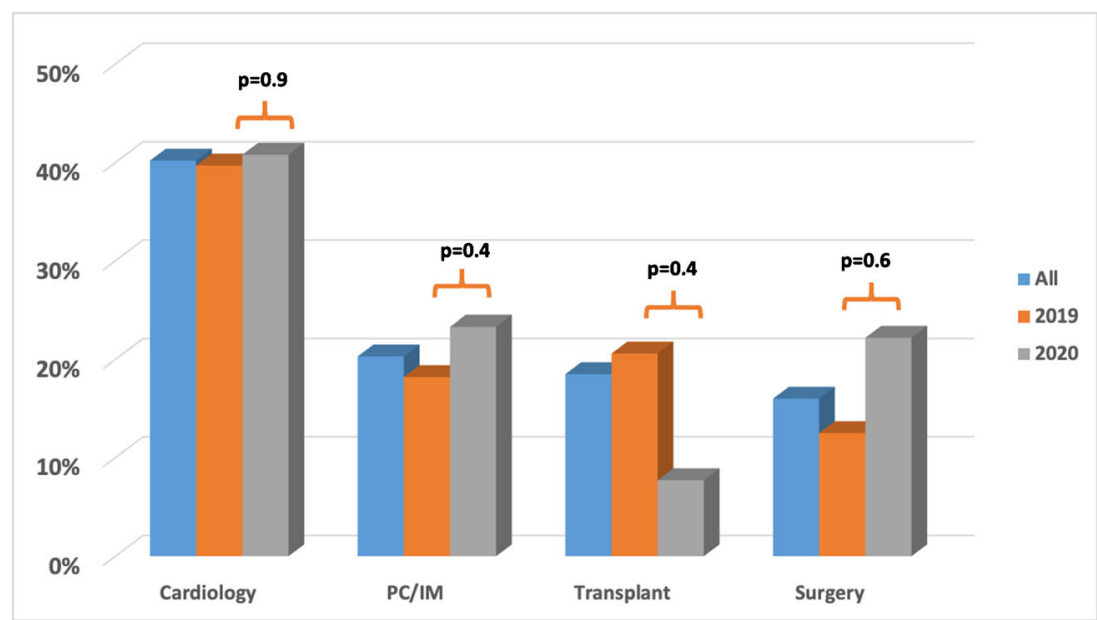


performed, it can be deduced that $68 \%$ of abnormal tests were missed during the pandemic. A similar calculation for our center demonstrates that we have missed $81 \%$ of abnormal studies during this period.

Our study is limited by the relatively small sample size, the retrospective design, and the inclusion of patients from a single medical center, which limits the generalizability of our findings. We used a historical control group rather than concurrent group with no restrictions on utilization of SPECTMPI due to feasibility. We note that the rate of abnormal SPECT-MPI is different at each medical center. In this regard, the rate of abnormal SPECT-MPI at our center is several folds higher than that described by Rozanski et al. and more similar to Duvall et al. [12, 35] and Nappi et al. [34] As such, selection of a historical national rather than center-specific control group would lead to erroneous conclusions.

In conclusion, during the COVID-19 pandemic, we experienced significant reductions in performance of SPECT-MPI. This reduction was accompanied by a shift of referred patients away from screening and towards those with symptoms. However, this did not translate into a higher yield of abnormal SPECT-MPI highlighting current limitations in predicting which patients will have abnormal studies.

Data availability All data was collected with IRB approval.

\section{Compliance with ethical standards}

Conflict of interest Dr. Hage reports investigator-initiated grant support from Astellas Pharma. All other authors declare that they have no conflict of interest.

Ethics approval This study has IRB approval from the University of Alabama at Birmingham.

\section{Consent to participate N/A}

Consent for publication N/A

Code availability N/A

\section{References}

1. Wiersinga WJ, Rhodes A, Cheng AC, Peacock SJ, Prescott HC. Pathophysiology, transmission, diagnosis, and treatment of coronavirus disease 2019 (COVID-19): a review. JAMA. Published online July 10, 2020. https://doi.org/10.1001/jama.2020.12839.

2. Dong E, Du H, Gardner L. An interactive web-based dashboard to track COVID-19 in real time. Lancet Infect Dis; published online Feb 19. https://doi.org/10.1016/S1473-3099(20)30120-1.

3. Cases in the U.S. (n.d.). Retrieved November 10, 2020, from https://www.cdc.gov/coronavirus/2019-ncov/cases-updates/casesin-us.html

4. Diaz A, Sarac BA, Schoenbrunner AR, Janis JE, Pawlik TM. Elective surgery in the time of COVID-19. Am J Surg.
2020;219(6):900-2. https://doi.org/10.1016/j.amjsurg.2020.04. 014.

5. Naidich JJ, Boltyenkov A, Wang JJ, Chusid J, Hughes D, Sanelli PC. Impact of the coronavirus disease 2019 (COVID-19) pandemic on imaging case volumes. J Am Coll Radiol. 2020;17(7):865-72. https://doi.org/10.1016/j.jacr.2020.05.004.

6. Garcia S, Albaghdadi MS, Meraj PM, et al. Reduction in STsegment elevation cardiac catheterization laboratory activations in the United States during COVID-19 pandemic. J Am Coll Cardiol. 2020;75(22):2871-2. https://doi.org/10.1016/j.jacc.2020.04.011.

7. Kadavath S, Mohan J, Ashraf S, et al. Cardiac catheterization laboratory volume changes during COVID-19-findings from a cardiovascular fellows consortium [published online ahead of print, 2020 Jun 11]. Am J Cardiol. 2020;130:168-9. https://doi.org/10.1016/j. amjcard.2020.06.009.

8. De Rosa S, Spaccarotella C, Basso C, et al. Reduction of hospitalizations for myocardial infarction in Italy in the COVID-19 era. Eur Heart J. 2020;41(22):2083-8. https://doi.org/10.1093/eurheartj/ ehaa409.

9. Mafham MM, Spata E, Goldacre R, et al. COVID-19 pandemic and admission rates for and management of acute coronary syndromes in England. Lancet. 2020;396(10248):381-9. https://doi.org/10. 1016/S0140-6736(20)31356-8.

10. Skali H, Murthy VL, Al-Mallah MH, et al. Guidance and best practices for nuclear cardiology laboratories during the coronavirus disease 2019 (COVID-19) pandemic: an information statement from ASNC and SNMMI. J Nucl Cardiol. 2020;27(3):1022-9. https://doi.org/10.1007/s12350-020-02123-2.

11. Skali H, Murthy VL, Paez D, et al. Guidance and best practices for reestablishment of non-emergent care in nuclear cardiology laboratories during the coronavirus disease 2019 (COVID-19) pandemic: an information statement from ASNC, IAEA, and SNMMI : endorsed by the Infectious Diseases Society of America [published online ahead of print, $2020 \mathrm{Jul}$ 24]. J Nucl Cardiol. 2020:1-8. https://doi.org/10.1007/s12350-020-02203-3.

12. Rozanski A, Gransar H, Hayes SW, et al. Temporal trends in the frequency of inducible myocardial ischemia during cardiac stress testing: 1991 to 2009. J Am Coll Cardiol. 2013;61(10):1054-65. https://doi.org/10.1016/j.jacc.2012.11.056.

13. Duvall WL, Rai M, Ahlberg AW, O'Sullivan DM, Henzlova MJ. A multi-center assessment of the temporal trends in myocardial perfusion imaging. J Nucl Cardiol. 2015;22(3):539-51. https://doi.org/ 10.1007/s12350-014-0051-X.

14. Iskandrian AE, Hage FG. Declining frequency of ischemia detection using stress myocardial perfusion imaging. J Am Coll Cardiol. 2013;61(10):1066-8. https://doi.org/10.1016/j.jacc.2012.12.009.

15. Elgendy IY, Mahmoud A, Shuster JJ, Doukky R, Winchester DE. Outcomes after inappropriate nuclear myocardial perfusion imaging: a meta-analysis. J Nucl Cardiol. 2016 Aug;23(4):680-9. doi: https://doi.org/10.1007/s12350-015-0240-2

16. Doukky R, Hayes K, Frogge N. Are cardiologists truly better at appropriately selecting patients for stress myocardial perfusion imaging? Int J Cardiol. 2014 Sep;176(1):285-6. https://doi.org/10. 1016/j.ijcard.2014.06.087.

17. Kuehn BM. Telemedicine helps cardiologists extend their reach. Circulation. 2016 Oct 18;134(16):1189-91. https://doi.org/10. 1161/CIRCULATIONAHA.116.025282.

18. De Simone V, Guarise P, Guardalben S, et al. Telecardiology during the Covid-19 pandemic: past mistakes and future hopes. Am $J$ Cardiovasc Dis. 2020;10(2):34-47. Published 2020 Jun 15.

19. Henzlova MJ, Cerqueira MD, Mahmarian JJ, Yao SS. Quality Assurance Committee of the American Society of Nuclear Cardiology. Stress protocols and tracers. J Nucl Cardiol. 2006;13(6):e80-90. https://doi.org/10.1016/j.nuclcard.2006.08. 011. 
20. Hage FG, Dean P, Iqbal F, Heo J, Iskandrian AE. A blunted heart rate response to regadenoson is an independent prognostic indicator in patients undergoing myocardial perfusion imaging. J Nucl Cardiol. 2011;18(6):1086-94. https://doi.org/10.1007/s12350011-9429-1.

21. Hage FG, Ghimire G, Lester D, et al. The prognostic value of regadenoson myocardial perfusion imaging. J Nucl Cardiol. 2015;22(6):1214-21. https://doi.org/10.1007/s12350-014-0050-y.

22. Ives CW, AlJaroudi WA, Kumar V, et al. Prognostic value of myocardial perfusion imaging performed pre-renal transplantation: post-transplantation follow-up and outcomes. Eur J Nucl Med Mol Imaging. 2018;45(11):1998-2008. https://doi.org/10.1007/ s00259-018-4068-2.

23. Colon CM, Marshell RL, Roth CP, Farag AA, Iskandrian AE, Hage FG. The prognostic value of myocardial perfusion imaging in patients with type 2 myocardial infarction [published online ahead of print, 2019 Oct 23]. J Nucl Cardiol. 2019; https://doi.org/10.1007/ s12350-019-01915-5. doi:https://doi.org/10.1007/s12350-01901915-5

24. McRee CW, Brice LR, Farag AA, Iskandrian AE, Hage FG. Evolution of symptoms in patients with stable angina after normal regadenoson myocardial perfusion imaging: the Radionuclide Imaging and Symptomatic Evolution study (RISE) [published online ahead of print, 2020 Aug 4]. J Nucl Cardiol. 2020; https://doi. org/10.1007/s12350-020-02298-8. doi:https://doi.org/10.1007/ s12350-020-02298-8

25. Ficaro EP, Lee BC, Kritzman JN, Corbett JR. Corridor4DM: the Michigan method for quantitative nuclear cardiology. J Nucl Cardiol. 2007;14(4):455-65. https://doi.org/10.1016/j.nuclcard. 2007.06.006.

26. Germano G, Kiat H, Kavanagh PB, et al. Automatic quantification of ejection fraction from gated myocardial perfusion SPECT. J Nucl Med. 1995;36(11):2138-47.

27. Zoghbi WA, DiCarli MF, Blankstein R, et al. Multimodality cardiovascular imaging in the midst of the COVID-19 pandemic: ramping up safely to a new normal. JACC Cardiovasc Imaging. 2020;13(7):1615-26. https://doi.org/10.1016/j.jcmg.2020.06.001.

28. Farag AA, AlJaroudi W, Neill J, et al. Prognostic value of silent myocardial infarction in patients with chronic kidney disease being evaluated for kidney transplantation. Int J Cardiol. 2017;249:37782. https://doi.org/10.1016/j.ijcard.2017.09.175.

29. Andrikopoulou E, Hage FG. Heart rate response to regadenoson: making the case for its value in clinical practice. J Nucl Cardiol. 2016;23(3):575-80. https://doi.org/10.1007/s12350-015-0269-2.

30. Bajaj NS, Singh S, Farag A, et al. The prognostic value of nonperfusion variables obtained during vasodilator stress myocardial perfusion imaging. J Nucl Cardiol. 2016;23(3):390-413. https:// doi.org/10.1007/s12350-016-0441-3.

31. Megna R, Assante R, Zampella E, et al. Pretest models for predicting abnormal stress single-photon emission computed tomography myocardial perfusion imaging [published online ahead of print, 2019 Nov 11]. J Nucl Cardiol. 2019;https://doi.org/10. 1007/s12350-019-01941-3. doi:https://doi.org/10.1007/s12350019-01941-3

32. Dunn TS 2nd, Hage FG. Stress myocardial perfusion imaging: can we tell the results without doing the test? [published online ahead of print, 2020 May 6]. J Nucl Cardiol. 2020;https://doi.org/10.1007/ s12350-020-02133-0. doi:https://doi.org/10.1007/s12350-02002133-0

33. Duvall WL, Baber U, Levine EJ, Croft LB, Henzlova MJ. A model for the prediction of a successful stress-first Tc-99m SPECT MPI. J Nucl Cardiol. 2012;19(6):1124-34. https://doi.org/10.1007/ s12350-012-9606-x.

34. Nappi C, Megna R, Acampa W, et al. Effects of the COVID-19 pandemic on myocardial perfusion imaging for ischemic heart disease [published online ahead of print, 2020 Aug 11]. Eur J Nucl Med Mol Imaging. 2020:1-7. https://doi.org/10.1007/s00259-02004994-6.

35. Duvall WL, Rai M, Ahlberg AW, O'Sullivan DM, Henzlova MJ. A multi-center assessment of the temporal trends in myocardial perfusion imaging. J Nucl Cardiol. 2015;22(3):539-51. https://doi.org/ 10.1007/s12350-014-0051-x.

Publisher's note Springer Nature remains neutral with regard to jurisdictional claims in published maps and institutional affiliations. 